\title{
Vonoprazan May Provide Better Results than PPIs in Helicobacter Pylori Eradication and Beyond - Is it Time for a Change?
}

\author{
Matteo Ghisa $^{1}$, Brigida Barberio ${ }^{1}$, Vincenzo Savarino ${ }^{2}$, Edoardo Savarino ${ }^{1}$
}

1) Department of

Surgery, Oncology and

Gastroenterology, University

of Padova, Padova

2) Department of Internal

Medicine, University of

Genova,

Genova, Italy

\author{
Address for correspondence: \\ Edoardo Savarino \\ Gastroenterology \\ Unit, Department of \\ Surgery, Oncology and \\ Gastroenterology \\ University of Padova \\ Via Giustiniani 2, 351258 \\ Padova, Italy \\ edoardo.savarino@unipd.it
}

Received: 07.11.2019

Accepted: 10.11.2019
Vonoprazan (VPZ) is a firstin-class potassium-competitive acid blocker (P-CAB) able to inhibit acid secretion by competitively blocking availability of potassium to hydrogen-potassium ATPase. In pharmacokinetic terms, it has numerous advantages over proton pump inhibitors (PPIs), which present a slower onset of action, CYP450-depending metabolism, instability in acidic conditions and incomplete efficacy on the suppression of nocturnal acid production. Moreover, unlike PPIs, the VPZ absorption rate is not affected by food and the drug can be taken independently from mealtimes $[1,2]$. From a pharmacodynamic point of view, VPZ is quickly absorbed and reaches high concentrations in the parietal cells immediately after the first consumption, while PPIs require 3-5 days to reach the steady state inhibition of acid secretion. This rapid action allows the use of these drugs also on-demand [1].

The spectrum of VPZ indications has expanded overtime and nowadays it is similar to that of PPIs, including gastroesophageal reflux disease (GERD), gastric and duodenal ulcers healing, management of upper gastrointestinal (GI) bleeding, non-steroidal antiinflammatory drugs (NSAIDs) - associated ulcers, Helicobacter pylori (H. pylori) eradication therapy and artificial ulcers induced by endoscopic submucosal dissection (ESD) $[1,3,4]$. As to the treatment of GERD, VPZ has been shown to be equally effective as PPIs in various studies using different dosages $(5-40 \mathrm{mg})[5,6]$. The non-inferiority of VPZ compared to lansoprazole (LPZ) was also confirmed in the treatment and prevention of the gastric ulcer [7]. Studies with VPZ (both 10 and $20 \mathrm{mg}$ ) showed similar results to those previously obtained with PPIs, in patients taking both NSAIDs and long-term low-dose aspirin, in the absence of severe adverse effects $[8,9]$. In addition, a recent systematic review and meta-analysis of 12 studies ( $\mathrm{n}=1265$ patients) comparing VPZ and PPIs in the treatment of ESD-induced artificial ulcers and in the prevention of delayed bleeding, showed a significantly higher healing rate at 4 weeks post-ESD in the VPZ group than in the PPI group (RR 1.20) [10].

In this issue of the journal, Shinmura et al. [11] performed a large retrospective cohort study comparing the efficacy of a tailored VPZ-based triple therapy with that of a VPZ-based conventional regimen in patients with $H$. pylori infection. These authors included 920 patients with $H$. pylori infection: out of them 541, who did not undergo a bacterial susceptibility test, received conventional therapy [VPZ plus clarithromycin (CAM) and amoxicillin (AMX)] and 379, who underwent a susceptibility test, received a tailored therapy [VPZ with CAM and AMX in the CAM sensitive (CAM-S) patients and VPZ with metronidazole (MNZ) and AMX in the CAM resistant (CAM-R) patients]. In the group of patients receiving conventional therapy, 165 had CAM-S bacteria and 123 had CAM-R bacteria, while in the group of patients receiving tailored therapy $(\mathrm{n}=379), 215$ had CAM-S bacteria and received CAM-containing triple therapy, while 164 had CAM-R bacteria and received MNZ-containing triple therapy. The eradication rates of tailored therapy by both intent-totreat (ITT) and per protocol (PPS) analyses were $90.0 \%$ and $96.3 \%$, respectively, which were significantly higher than those of conventional therapy $(85.0 \%$ and $90.2 \%, p=0.0028$ and $\mathrm{p}=0.0008$, respectively). Using the conventional therapy, the eradication rate of patients with CAM-R bacteria (85.8\%) was significantly lower $(\mathrm{p}<0.05)$ than that of those with CAM-S (93.2\%). Using the tailored therapy, the eradication rate of patients with CAM-R bacteria (98.1\%) did not differ from that of those with CAM-S bacteria (95.0\%) and was significantly increased compared to that of cases with CAM-R bacteria in the conventional therapy group $(p=0.0002)$. In patients with 
CAM-S bacteria, there was no difference in the eradication rate between conventional and tailored therapies. In the conventional therapy group, the eradication rate of patients with CAM-R and AMX-R bacteria (65\%) was significantly lower than that of patients with CAM-S and AMX-S bacteria $(p=0.001)$. By univariate analysis, patients with AMX-R and CAM-R, who used conventional therapy had a high risk factor for eradication failure $(\mathrm{OR}=2.608, \mathrm{p}=0.017$, and $\mathrm{OR}=2.839, \mathrm{p}=0.0008$, respectively). By multivariate analysis, only conventional therapy was detected as a risk factor for the failure of VPZ-based triple therapy $(\mathrm{OR}=3.113, \mathrm{p}=0.0005)$.

This is the first report comparing the efficacy of VPZ-based tailored triple therapy to VPZ-based conventional therapy and it demonstrated that the former is more effective in the eradication of $H$. pylori than the latter, according to bacterial antibiotic susceptibility. However, it must be acknowledged that this study has a relevant limitation represented by its retrospective design and therefore, in order to generalize these results, future prospective trials are required.

Numerous studies on the use of VPZ-based eradication regimens have been carried out since 2014, when VPZ was approved as both first- and second-line therapy for the treatment of $H$. pylori infection in Japan. Murakami et al. [12] were the first to compare VPZ $20 \mathrm{mg}$ bid and LPZ $30 \mathrm{mg}$ bid, given as a 7-day AMX and CAM first-line triple therapy. In their randomized study, 650 patients were enrolled and the eradication rates were $92.6 \%$ with VPZ versus $75.9 \%$ with LPZ $(\mathrm{p}<0.0001)$. Subclassifying patients in CAM-S and CAM-R, VPZ response rates were $100 \%$ and $87.5 \%$, respectively, while they were significantly reduced with LPZ to $88 \%$ and $53.8 \%$, respectively. The overall superiority of VPZ-based therapy vs the PPI-based one for the eradication of $H$. pylori infection was confirmed in many subsequent studies [13-17]. However, it clearly emerged from many of them that the success of VPZbased schemes was not universal and depended on both the CAM sensitivity and the number of treatment attempts (first or second) [1, 15-21].

Stratifying patients on the basis of CAM sensitivity, the superiority of VPZ-based regimens as a first-line triple therapy vs the PPI-based ones in CAM-R patients has been documented in various studies and, accordingly, was recently confirmed by two meta-analyses $[16,17]$. On the contrary, no significant difference in the eradication rate between VPZand PPI-based regimens was observed in CAM-S patients [1, 15, 18-20]. In particular, Dong et al. [16] evaluated 14 studies ( $n=14,636$ patients) in a meta-analysis and found that VPZ was not superior to PPIs as part of a second-line triple therapy containing $\mathrm{MNZ}$ on both intention-to-treat $(83.4 \%$ vs $82 \%, p=0.79)$ and per-protocol analyses $(89.3 \%$ vs $90.1 \%$, $\mathrm{p}=0.06$ ) [16]. This seems to suggest that eradication regimens containing MNZ probably do not benefit from the strong suppression of gastric acid secretion achievable with VPZ. As to the safety of VPZ, Jung et al. [17] elaborated data from 10 studies including 10,644 patients and showed that there was no difference in terms of any adverse events between VPZ- and PPI-based regimens (pooled RR [95\% CI] $=1.02$ [0.78-1.34]).

To date, the results reported in the medical literature show that there is a consistent body of studies comparing PPI and VPZ against $H$. pylori infection and they show a substantial similarity of these two components of the tested triple therapies, when the variable CAM sensitivity was not taken into consideration. Therefore, the selection of the P-CAB seems to depend on the evaluation of its cost effectiveness and the subtype of the clinical scenario [22]. The study by Shinmura et al. [11], on the contrary, shows the superiority of VPZbased eradication therapy, providing that patients undergo the analysis of their CAM susceptibility. In fact, the eradication rate of patients with CAM-R bacteria rose from $85.8 \%$ obtained with the conventional VPZ-based triple regimen to $98.1 \%$ with the VPZ-based tailored one. Therefore, the preventive classification of patients on the basis of CAM sensitivity appears to be fundamental for performing a successful VPZ-based tailored therapy, even though the above excellent results must be confirmed in prospective clinical studies.

In conclusion, VPZ represents a valid alternative to PPIs in a wide spectrum of acid-related clinical situations because of its strong acid suppression and rapid onset of action. As an antisecretory agent associated with antibiotics for the eradication of $H$. pylori infection, VPZ-based schemes seem to be able to obtain excellent eradication rates, when they are used as first-line therapy and in regions where the resistance to the traditional antibiotics, in particular CAM, is high. The success of this VPZ-based triple therapy tailored according to bacterial antibiotic susceptibility has been observed in a large, but retrospective study and must be confirmed in future prospective randomized clinical trials evaluating CAM sensitivity.

Conflict of interests: None to declare.

Authors' contribution: M.G., B.B.: analyzed the data and drafted the manuscript. V.S., E.S.: wrote the paper. E.S. critically revised the paper. All the authors approved the final version.

\section{REFERENCES}

1. Graham DY, Dore MP. Update on the Use of Vonoprazan: A Competitive Acid Blocker. Gastroenterology 2018;154:462-466. doi:10.1053/j. gastro.2018.01.018

2. Martinucci I, Blandizzi C, Bodini G, et al. Vonoprazan fumarate for the management of acid-related diseases. Expert Opin Pharmacother 2017;18:1145-1152. doi:10.1080/14656566.2017.1346087

3. Savarino E, Martinucci I, Furnari M, et al. Vonoprazan for treatment of gastroesophageal reflux: pharmacodynamic and pharmacokinetic considerations. Expert Opin Drug Metab Toxicol 2016;12:1333-1341. doi:10.1080/17425255.2016.1214714

4. Shimozato A, Sasaki M, Ogasawara N, et al. Risk factors for delayed ulcer healing after endoscopic submucosal dissection of gastric neoplasms. J Gastrointestin Liver Dis 2017;26:363-368. doi:10.15403/ jgld.2014.1121.264.kas

5. Oshima T, Miwa H. Potent Potassium-competitive Acid Blockers: A New Era for the Treatment of Acid-related Diseases. J Neurogastroenterol Motil 2018;24:334-344. doi:10.5056/jnm18029

6. Xiao Y, Zhang S, Dai N, et al. Phase III, randomised, double-blind, multicentre study to evaluate the efficacy and safety of vonoprazan compared with lansoprazole in Asian patients with erosive oesophagitis. Gut 2019 Aug 13. doi:10.1136/gutjnl-2019-318365 